\section{AB0691 ANALYSIS OF SARS-COV-2 ANTIBODIES IN NON- COVID-19 PATIENTS: COMPARISON BETWEEN SYSTEMIC SCLEROSIS PATIENTS AND HEALTHY CONTROLS}

L. Giardullo ${ }^{1}$, C. Rotondo ${ }^{1}$, A. Corrado ${ }^{1}$, N. Maruotti ${ }^{1}$, R. Colia ${ }^{1}$, A. Altomare ${ }^{1}$, E. Sanpaolo ${ }^{1}$, D. Cici ${ }^{1}$, F. P. Cantatore ${ }^{1} .{ }^{1}$ University of Foggia "Policlinico Riuniti", Medical and Surgical Sciences, Rheumatology Clinic "Mario Carrozzo", Foggia, Italy

Background: Previous study evidenced a cross-reactivity between Sars-Cov-2 antibodies and autoimmune tissue antigen involved in connective tissue diseases, as nuclear antigen (NA), extractable nuclear antigen (ENA), histone and collagen (1). No study has been published about the titer of Sars-Cov-2 antibodies in non-infected patients with autoimmune disease.

Objectives: To evaluate the titer of SARS-CoV-2 antibodies in non-COVID-19 patients and compare it between systemic sclerosis (SSc) patients and healthy controls $(\mathrm{HC})$.

Methods: A total of 58 patients with SSc (who fulfilled ACR/EULAR 2013 SSc classification criteria) and $18 \mathrm{HC}$ were enrolled. Sera of all participants were collected, and SARS-CoV-2 antibodies (IgG and IgM) were evaluated by means ELISA. In all participants swabs for SARS-CoV-2 by real-time reverse-transcriptase-polymerase-chain-reaction assay were reported negative. Demographic, clinical, and autoimmune serological characteristics of SSc patients were recorded. The normal distribution was assessed using the Shapiro-Wilk's test. Exclusion criteria was previous or actual Sars-Cov-2 infection. Comparisons between study groups of patients were evaluated by the Student's t-test or Mann - Whitney U-test as appropriate. The differences between categorial variables were assessed by Pearson chi-square or Fisher's exact test, as opportune. Statistical significance was set at $p \leq 0.05$.

Results: We observed significant differences between SSc patients and $\mathrm{HC}$ in serum levels of Sars-Cov-2 antibodies (IgG: $1,4 \pm 2,1 \mathrm{AU} / \mathrm{ml}$ vs $0,36 \pm 0,19 \mathrm{AU} / \mathrm{ml}$ respectively ( $p=0,001)$; and lgM: $2,5 \pm 3,1 \mathrm{AU} / \mathrm{ml}$ vs $0,8 \pm 0,7 \mathrm{AU} / \mathrm{ml}(\mathrm{p}=0,022))$. In 5 SSc patients was found titer of Sars-Cov-2 antibodies (IgG) exceeding the cut-off, but the control of swabs for SARS-CoV-2 by real-time reverse-transcriptase-polymerase-chain-reaction assay were negative. No significative differences in SarsCov-2 autoantibodies titer were found in subgroup of SSc patients with or without ILD or PAH, limited or diffuse skin subset, and different autoantibodies profile. Furthermore, antibodies titer was not associated with different drugs (steroid, methotrexate, mofetil-mycophenolate and bosentan) in use.

Conclusion: A cross mimicking between Sars-Cov-2 antibodies and antinuclear antibodies or anti ENA could be hypothesized. Further studies are necessary to unravel the reliability of Sars-Cov-2 antibodies detection in autoimmune disease. REFERENCES:

[1] Vojdani, A., Vojdani, E., \& Kharrazian, D. (2021). Reaction of human monoclonal antibodies to SARS-CoV-2 proteins with tissue antigens: Implications for autoimmune diseases. Frontiers in Immunology, 11, 3679

Disclosure of Interests: None declared

DOI: 10.1136/annrheumdis-2021-eular.3143

\section{AB0692 REACTIVE ARTHRITIS ASSOCIATED WITH COVID-19 INFECTION: A REVIEW}

F. Alkindi', K. Alseiari ${ }^{2}$, K. Al Naqbi ${ }^{3}{ }^{1}$ Tawam Hospital, Internal Medicine, Al Ain, United Arab Emirates; ${ }^{2}$ Tawam Hospital, Internal Medicine, AlAin, United Arab Emirates: ${ }^{3}$ Tawam Hospital, Rheumatology Department, AlAin, United Arab Emirates

Background: The incidence of COVID-19 infection is increasing globally with high mortality rate. Cytokine release syndrome might contribute to extra-pulmonary manifestations such as acute kidney injury, venous thrombosis, neurological complications, hepatic and myocardial injury. Reactive arthritis is an emerging musculoskeletal (MSK) manifestation post COVID-19 infection. Reactive arthritis manifests as asymmetrical, oligoarthritis mainly involving peripheral or axial joints of lower extremities and associated with extra-articular manifestations.

Objectives: review the clinical presentation and management outcomes of COVID-19 associated reactive arthritis.

Methods: A literature research was conducted using PubMed and Google scholar for published abstracts, case reports, and studies from January 2020 to January 2021. We used search keywords" reactive arthritis", "COVID-19 pneumonia," "SARS CoV2 infection", and "Musculoskeletal." Descriptive analysis was used due to small sample size.

Results: COVID-19 associated reactive arthritis is rarely reported. In review of literature, 10 cases were identified and we included our case of hip arthritis and avascular necrosis post COVID-19 infection. The mean age of cases $(n=11)$ was of 42.8 years and $54.5 \%$ of patients were males. (Table 1) The median duration of reactive arthritis diagnosis from COVID-19 infection ranged from 1 to 8 weeks. The severity of COVID-19 infection varied from mild $(n=6)$ to severe disease $(n=2)$ per description in each reported case. Majority of the patients had oligoarticular involvement (2 to 4 joints) $45.5 \%$, followed by monoarticular $36.4 \%$, and polyarticular (> 4 joints) in $18 \%$. Extra-articular manifestations were identified in $54.5 \%$ of patients including skin rash (erythematous itchy rash), urticarial rash, wrist tendinitis, Achilles enthesitis /tendonitis and balanitis. HLA-B27 testing was done in five patients and only one patient had a positive result. Plain radiographs were normal. Therapy provided of such cases were NSAIDS $(n=4)$, steroids (oral, intra-articular) $(n=1)$ or combination of steroids and NSAIDS $(n=3)$ with favorable outcomes. The median reported follow up period ranged from 1.5 to 8 weeks.

Table 1. Characteristics of the reported reactive arthritis cases post COVID-19 infection

\begin{tabular}{|c|c|}
\hline Clinical data of patients $(n=11)$ & Results $(n=11)$ \\
\hline Age (mean) & 42.8 Years old \\
\hline \multicolumn{2}{|l|}{ Sex \% } \\
\hline & Female $(n=5[45.5 \%])$ \\
\hline & Male $(n=6[54.5 \%])$ \\
\hline \multirow[t]{3}{*}{ Severity of COVID-19 infection } & Mild $(n=6[54.5 \%])$ \\
\hline & Moderate $(n=3[27.3 \%])$ \\
\hline & Severe $(n=2[18 \%])$ \\
\hline Prior history of rheumatologic disease & $(n=0)$ \\
\hline Time to onset of reactive arthritis (median) & 1 to 8 weeks \\
\hline \multirow{3}{*}{$\begin{array}{l}\text { Number of joints involved at time of } \\
\text { diagnosis }\end{array}$} & Monoarticular $(n=4[36.4 \%])$ \\
\hline & Oligoarticular $(n=5[45.5 \%])$ \\
\hline & Polyarticular $(n=2[18 \%])$ \\
\hline \multirow{3}{*}{$\begin{array}{l}\text { Location of involved joints (upper vs. lower } \\
\text { limbs) }\end{array}$} & Upper joints (18\%) \\
\hline & Lower joints $(54.5 \%)$ \\
\hline & Both $(27.3 \%)$ \\
\hline Enthesitis & $(n=1[9 \%])$ Achilles \\
\hline Tendonitis & $(\mathrm{n}=2[18 \%])$ (wrist $/$ Achilles tendonitis) \\
\hline $\begin{array}{l}\text { Extra-articular involvement (eyes, skin, GI, } \\
\text { GU, others) }\end{array}$ & $\begin{array}{c}\text { - Skin rash ( } \mathrm{n}=2[18 \%]) \text { (erythematous itchy } \\
\text { rash, urticarial rash) }\end{array}$ \\
\hline Inflammatory back pain & $\begin{array}{l}\text { - GU: balanitis }(\mathrm{n}=1 \text { [9\%]) } \\
(\mathrm{n}=0)\end{array}$ \\
\hline Avascular necrosis of bone & $(n=1[9 \%])$ \\
\hline HLA-B27 & $(n=1[9 \%]),(n=5$ tested $)$ \\
\hline Autoimmune workup & Negative \\
\hline \multirow[t]{5}{*}{ Treatment of reactive arthritis: } & DMARDs $(\mathrm{n}=0)$ \\
\hline & NSAIDs $(n=4[36.4 \%])$ \\
\hline & Steroids $(n=1[9 \%])$ \\
\hline & NSAIDs and steroids $(n=3[27.3 \%])$ \\
\hline & Not provided $(n=2)$, others $(n=1)$ \\
\hline Outcomes of MSK symptoms & Improvement $(\mathrm{n}=11[100 \%])$ \\
\hline Follow up period (Median, weeks) & 1.5 to 8 weeks \\
\hline
\end{tabular}

Gastrointestinal (GI), genitourinary (GU), NSAIDs: non-steroidal anti-inflammatory drug

Conclusion: Reactive arthritis is a rare MSK manifestations post COVID-19 infection. HLA-B27 positive testing might indicate severe and delayed form of arthritis with risk of recurrence. Larger studies are required to delineate the potential risk factors and long-term management outcomes for reactive arthritis associated with COVID-19 infection.

REFERENCES:

[1] Ono K, Kishimoto M, Shimasaki T, et al. Reactive arthritis after COVID-19 infection, RMD Open 2020;6:e001350. doi: 10.1136/rmdopen-2020-001350

Disclosure of Interests: None declared

DOI: 10.1136/annrheumdis-2021-eular.3174

\begin{tabular}{|l|l}
\hline AB0693 & NON-STEROIDAL ANTI-INFLAMMATORY DRUGS \\
& DURING CORONAVIRUS 19 PANDEMIC: WHAT DO \\
& TUNISIAN RHEUMATOLOGISTS THINK?
\end{tabular}

H. Ferjani ${ }^{1}$, H. Boussaa ${ }^{1}$, K. Maatallah ${ }^{1}$, H. Khalfalli ${ }^{1}$, D. Kaffel ${ }^{1}$, W. Hamdi ${ }^{1}$. ${ }^{1}$ Mohamed Kassab Institute of Orthopedics, Rheumatology, La Manouba, Tunisia

Background: Concerns over the safety of non-steroidal anti-inflammatory drugs (NSAIDs) use during severe acute respiratory syndrome associated with coronavirus 19 disease (covid-19) have raised. NSAIDs are one of the most commonly prescribed and used pain medications for acute and chronic rheumatic diseases such as spondyloarthritis (SpA) and osteoarthritis.

Objectives: This study aimed to assess the impact of covid-19 pandemic on NSAIDs prescription

Methods: A cross-sectional web survey was disseminated to all Tunisian rheumatologists through a mailing system and social media. The French version was accessible on Google form. It included close-ended questions about the prescription of NSAIDs during covid-19 pandemic. Participation was anonymous Data collection and analysis was performed between January the first and January 30, 2021.

Results: Among one hundred and thirty Tunisian rheumatologists, thirty responded to the online questionnaire. Ninety percent of participants were women. The mean age of rheumatologists was 34 years [25-57]. The duration of 
practice was inferior to 5 years in $59.3 \%$, between 5 and 10 years in $18.5 \%$, and superior to 10 years in $22.2 \%$ of cases. Sixty-three percent of rheumatologists reported that their activity decreased during covid-19 pandemic. NSAIDs prescription was avoided as much as possible in $40.7 \%$ of cases.

The participants indicated NSAIDs less frequently in $33.3 \%$ of cases, and as much as before the pandemic in one-quarter of cases.

Rheumatologists believed that NSAIDs worsen the respiratory symptoms (67\%), delay recovery (55\%), and increase mortality (48\%), hospitalization in intensive care (44\%), and infectious complications (33\%).

The participants suggested that the most incriminated NSAIDs were: Ibuprofen (7.4\%), indomethacin $(7.4 \%)$, celecoxib $(7.4 \%)$, and diclofenac $(3.4 \%)$. The majority of rheumatologists $(74 \%)$ believed that all NSAIDs had a similar risk. For patients with osteoarthritis, rheumatologists replaced NSAIDs with paracetamol and corticoids in $78 \%$ and $11 \%$ of cases, respectively. If mandatory, reducing NSAIDs doses or duration was an option in $22 \%$ and $74 \%$ of cases.

For patients with $\mathrm{SpA}$, half of rheumatologists did not change the treatment. However, the participants limited the use of NSAIDs or discontinued the treatment in patients with comorbidities. More than $60 \%$ of rheumatologists didn't know the effect of NSAIDs in the post-covid-19 syndrome.

Conclusion: Covid-19 pandemic has affected rheumatologists' practice. Rheumatic disease management during this pandemic may be challenging. More evidence is mandatory to standardize treatment prescription, especially with NSAIDs.

Disclosure of Interests: None declared

DOI: 10.1136/annrheumdis-2021-eular.3221

\section{AB0694 COVID-19 İNFECTION IN PATIENTS WITH RHEUMATIC DISEASES: TWO CENTERS EXPERIENCE}

\section{A. Sahahin ${ }^{1}$, M. A. Gedikli ${ }^{2}$, M. E. Derin ${ }^{3}$, B. Karakaş ${ }^{1}$, B. Karataş ${ }^{1}$, N. Çabuk} Çelik', İ. Yalçın' ${ }^{1}$ Sivas Cumhuriyet University, Internal Medicine Rheumatology, Sivas, Turkey; ${ }^{2}$ Sivas Cumhuriyet University, Internal Medicine, Sivas, Turkey; ${ }^{3}$ Sivas Numune Hospital, Internal Medicine - Rheumatology, Sivas, Turkey

Background: Coronavirus disease 2019 (COVID-19) is a new viral infection characterized by dry cough, fever, shortness of breath, fatigue and lymphopenia. It may be complicated by interstitial pneumonia leading to severe acute respiratory distress syndrome(1). The COVID-19 outbreak has increased concerns in those with rheumatological diseases(2).

Objectives: To present the clinical course of COVID-19 infection in patients with rheumatological disease and comorbid conditions of patients and drug use. Methods: 70 COVID-19 patients with autoimmune and autoinflammatory rheumatic diseases over the age of 18 who were followed up in the internal diseases-rheumatology department of Sivas Cumhuriyet University Hospital and Sivas Numune Hospital between April 2020 and January 2021 were included in this study. An application was made to the ethics committee of Sivas Cumhuriyet University Hospital and approval was obtained. Demographic data, comorbidities, smoking, treatments for their diseases, symptoms and hospitalization status of the patients were evaluated.

Results: The median age of the women patients was 48 years (min: 20-max: 73), 41 years in men (min: 21-max: 78$) .15$ patients (21.4\%) were male, 55 patients $(78.6 \%)$ were female. The most common rheumatic disease was rheumatoid arthritis (RA, 21, 30\%) followed by ankylosing spondylitis $(19,27.1 \%)$, familial Mediterranean fever (13, 18.6\%), Behçet's disease (12, 17.1\%). 22 (31.4\%) patients hydroxychloroquine sulfate, $14(20 \%)$ leflunomide, $7(10 \%)$ patients methotrexate, 6 (8.6\%) patients sulfasalazine, 10 (14.3\%) patients azathioprine/ mycophenolate mofetil, 22 (31.4\%) patients were using colchicine. As a biological medicine; $20(28.6 \%)$ patients were using infliximab, $5(7.1 \%)$ patients were using adalimumab, $6(8.6 \%)$ patients were using rituximab, and $4(5.7 \%)$ patients were using tocilizumab. Other are summarized in Table 1. The most common comorbidities were hypertension (25.7\%) and diabetes mellitus (DM 17.1\%). While 56 patients $(80 \%)$ had symptoms of COVID-19, 14 patients $(20 \%)$ were asymptomatic. The most common symptoms was fever $(32,45.7 \%)$ followed by cough $(29,41.4 \%)$, dyspnea $(28,40 \%)$, myalgia $(25,35.7 \%)$, loss of taste and smell $(18,25.7 \%) .21$ pneumonia cases were diagnosed. $22(31.4 \%)$ patients were hospitalized. Mortality rate was $2 / 70,2.9 \%$. Two RA patients who just treated with rituximab and tocilizumab were died. 10 cases were using $>10 \mathrm{mg} /$ day prednisolone, 2 died, 8 cases were hospitalized and had severe disease. They had hypertension and DM. Characteristics of the patients are summarized in Table 2. Conclusion: The course of COVID-19 in rheumatic diseases is not clearly. Meta-analysis has shown that the frequency of COVID-19 is increased in autoimmune diseases. However, this increase was mostly associated with the use of $>$ $10 \mathrm{mg} /$ day prednisolone. It has been stated that the use of biological drugs and Dmard causes the SARS-CoV 2 process with a milder course and therefore their use is safe (3). Similar results have been obtained in global registry registers (4). In our data, there was no increase in the mortality rate compared to the normal population. However, especially the use of rituximab may increase the rate of severe disease and mortality, so its use should be careful. There is a need for more comprehensive studies.

\section{REFERENCES:}

[1] Guan W, Ni Z, Hu Y, et al. Clinical characteristics of coronavirus disease 2019 in China. N Engl J Med 2020

[2] N. Chen, M. Zhou, X. Dong,et al., Epidemiological and clinical characteristics of 99 cases of 2019 novel coronavirus pneumonia in Wuhan, China: a descriptive study, Lancet 395 (10223) (2020) 507`513

[3] Akiyama S,Hamdeh S, Micic D, et al, Prevalence and clinical outcomes of COVID-19 in patients with autoimmune diseases: a systematic review and meta-analysis,Ann Rheum Dis 2020 Oct 13;annrheumdis-2020-218946.

[4] Gianfrancesco M, Hyrich KL, Al-Adely S,et al, Characteristics associated with hospitalisation for COVID-19 in people with rheumatic disease: data from the COVID-19 Global Rheumatology Alliance physician-reported registry. Ann Rheum Dis. 2020 Jul;79(7):859-866.

Disclosure of Interests: None declared

DOI: 10.1136/annrheumdis-2021-eular.3289

\section{AB0695 19 PATTERN OF COVID-19 IN PATIENTS WITH RHEUMATIC DISEASES UNDERGOING BIOLOGICAL THERAPY: A COHORT EXPERIENCE}

C. Ancuta ${ }^{1,2}$, C. Pomirleanu ${ }^{1,2}$, G. Strugariu ${ }^{2}$, L. Petrariu ${ }^{2}$, E. Ancuta ${ }^{3}$,

C. $\operatorname{Bran}^{4,5}$, R. Chirieac ${ }^{6}$, C. Mihailov ${ }^{7,8}$. ${ }^{1}$ University of Medicine and Pharmacy "GRIGORE T. POPA"IASI, Rheumatology, IASI, Romania; ${ }^{2}$ Clinical Rehabilitation Hospital, Rheumatology, IASI, Romania; " "Elena Doamna"Clinical Hospital, Research, IASI, Romania; ${ }^{4}$ Emergency Hospital "Sf loan", Rheumatology, IASI, Romania; ${ }^{5}$ University Stefan Cel Mare Suceava, Semiology and Immunology, Suceava, Romania; ${ }^{6}$ Sanocare Medical and Research Center, Rheumatology, IASI, Romania; ${ }^{7}$ Clinical CF Hospital of Constanta, Rheumatology, Constanta, Romania; ${ }^{8}$ Faculty of Medicine, "Ovidius" University Constanta, Internal Medicine and Rheumatology, Constanta, Romania

Background: Despite emerging vaccines, the world is in the midst of a coronavirus disease 2019 (COVID-19) pandemic. Outcomes of SARS-CoV2 infection remain a major concern in patients with rheumatic and musculoskeletal diseases, especially for those with uncontrolled disease.

Objectives: We aimed to investigate trends and outcomes of COVID-19 occurring in patients with chronic inflammatory rheumatic conditions treated with biologics and targeted synthetic disease modifying antirheumatic drugs (bDMARDs, tsDMARDs).

Methods: We included all confirmed cases of COVID-19 regardless of severity in patients with rheumatoid arthritis (RA), ankylosing spondylitis (AS) and psoriatic arthritis (PSA) undergoing bDMARDs or tsDMARDs treatment registered in our local COVID-19 reporting database. We collected relevant information about comorbidities, rheumatologic-related clinical activity (RAPID5, SDAI, BASDAI DAPSA), type of DMARD and glucocorticoid use, as well as COVID-19 related data as severity (ranging from asymptomatic to life-threatening forms), medication, hospitalization, intensive care unit admission, invasive mechanical ventilation and death.

We did a subgroup analysis among patients with a specific rheumatologic diagnosis, among different class of medication, patients who were or not hospitalized with COVID-19 looking how age, comorbidities, type of rheumatic condition and treatments impact COVID outcomes.

Results: 40 COVID-19 cases (positive PCR for SARS-CoV2) (4.67\%) were identified during the 6-month study period among 855 patients registered in our database of patients under biologic treatment, including 20 RA, 18 SpA, 2 PsA patients. The majority were in either low disease activity or remission, only two patients had active uncontrolled disease at the onset of coronavirus infection. 16 cases $(40 \%)$ were asymptomatic and were tested RT-PCR-positive during routine follow-ups for their disease, 13 cases (32.5\%) had mild and 8 cases (20\%) moderate illness; severe pneumonia and critical disease with acute respiratory distress syndrome were reported in only 3 cases, 2 recovered after; the only patients who died was 69 years old, had cardiac disease, hypertension and diabetes, had undertaken regular rituximab perfusion one month before coronavirus infection and developed pulmonary embolism followed by septic shock. Extreme fatigue was the dominant COVID-19 associated symptom apart from the classical ones including fever, cough, shortness of breath, sore throat, nasal congestion, headache, anosmia and ageusia myalgias and anorexia.

No specific pattern for patients requiring intensive care unit admission.

Conclusion: The COVID-16 infection rate in patients with inflammatory rheumatic disorders receiving biologics and tsDMARDs is pretty low; although immunosuppressed, these patients seem not to be at risk for severe COVID-19 illness and outcomes. These findings might reflect a potential protective role of certain biologics and/or JAK inhibitors for development and severity of COVID-19 in patients. 